\title{
Higher loop renormalization of a supersymmetric field theory
}

\author{
Marc P. Bellon ${ }^{\mathrm{a}, \mathrm{b}, 1}$, Gustavo Lozano ${ }^{\mathrm{c}}$, Fidel A. Schaposnik ${ }^{\mathrm{a}, \mathrm{b}, *, 2}$ \\ a CEFIMAS, Av. Santa Fe 1145, 1069 Capital Federal, Argentina \\ ${ }^{\mathrm{b}}$ Departamento de Física, Universidad Nacional de La Plata, C.C. 67, 1900 La Plata, Argentina \\ ${ }^{\mathrm{c}}$ Departamento de Física, FCEyN, Universidad de Buenos Aires, Pab. I, Ciudad Universitaria, 1428 Buenos Aires, Argentina
}

Received 19 March 2007; accepted 21 May 2007

Available online 24 May 2007

Editor: L. Alvarez-Gaumé

\begin{abstract}
Using Dyson-Schwinger equations within an approach developed by Broadhurst and Kreimer and the renormalization group, we show how high loop order of the renormalization group coefficients can be efficiently computed in a supersymmetric model. (c) 2007 Elsevier B.V. All rights reserved.
\end{abstract}

\section{Introduction}

Perturbative Quantum Field Theory is known for its tremendous successes, with its ability to obtain highly precise values in Quantum Electrodynamics, or the tests of the standard model involving radiative corrections to weak interactions. However, actual calculations become rapidly cumbersome and display a conjunction of analytical and combinatorial difficulties combined with the very fast growth of the number of relevant terms, which get compounded by the need to address the subtraction of the various subdivergencies.

In the quest of organizing principles for taming the combinatorial problem, a major progress has been the recognition of a Hopf algebra structure in the renormalization of Quantum Field Theories [1] and subsequent developments [2-4] and the link relating the cohomology of the introduced Hopf algebras with Dyson-Schwinger equations [5,6] (see [7] for a complete list of references). An application to the summation of a category of

\footnotetext{
* Corresponding author at: Departamento de Física, Universidad Nacional de La Plata, C.C. 67, 1900 La Plata, Argentina.

E-mail address: fidel@ fisica.unlp.edu.ar (F.A. Schaposnik).

1 On leave from, Laboratoire de Physique Théorique et Hautes Energies, Boite 126, 4 Place Jussieu, 75252 Paris Cedex 05. Unité Mixte de Recherche UMR 7589 Université Pierre et Marie Curie-Paris6; CNRS; Université Denis Diderot-Paris7.

2 Associate to CICBA.
}

diagrams in a simple Yukawa field theory or a $\phi^{3}$ theory in 6 dimension has been obtained by Broadhurst and Kreimer in [6]. In that work a very powerful method to compute higher order corrections to the anomalous dimension has been developed. Basically, it is based on a differential equation derived from the Dyson-Schwinger equation for the self-energy.

The purpose of this Letter is to extend such results to the case of a supersymmetric model. We have considered a WessZumino like model in which the non-renormalization of the vertex ensures that the order zero in a large $N$ expansion of the renormalization group functions can be obtained. We also expect that the known non-renormalization properties related to supersymmetry should allow to go beyond this initial category of diagrams in future works. Along the way, we propose an alternative derivation of the propagator-coupling duality presented in [6] based on intuitive renormalization group arguments.

\section{The model}

We consider massless chiral superfields $\Psi$ and $\Phi_{i}(i=$ $1,2, \ldots, N$ ) and their (antichiral) complex conjugates $\Psi^{+}$and $\Phi_{i}^{+}$, which satisfy the following constraints:

$$
\begin{aligned}
& \bar{D}_{\dot{\alpha}} \Psi=0, \quad \bar{D}_{\dot{\alpha}} \Phi_{i}=0, \\
& D_{\alpha} \Psi^{+}=0, \quad D_{\alpha} \Phi_{i}^{+}=0 .
\end{aligned}
$$




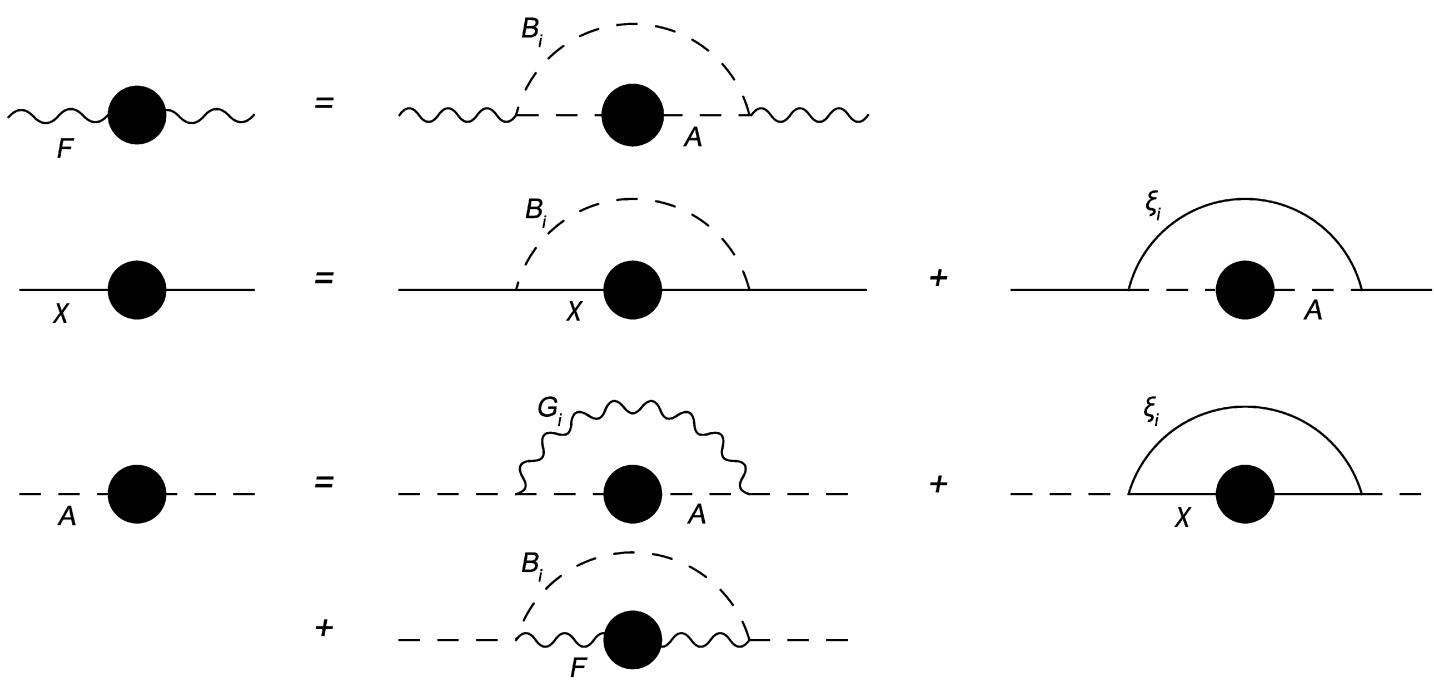

Fig. 1. Schwinger-Dyson equations.

The derivatives are defined as:

$D_{\alpha}=\frac{\partial}{\partial \theta^{\alpha}}+2 i \sigma_{\alpha \dot{\alpha}}^{\mu} \bar{\theta}^{\dot{\alpha}} \frac{\partial}{\partial y^{\mu}}, \quad \bar{D}_{\dot{\alpha}}=-\frac{\partial}{\partial \bar{\theta}^{\dot{\alpha}}}$

in terms of the chiral coordinates $y$ defined as

$y^{\mu}=x^{\mu}+i \theta \sigma^{\mu} \bar{\theta}$.

Each chiral superfield represents a complex scalar $\left(A, B_{i}\right)$, a Weyl fermion $\left(\chi, \xi_{i}\right)$ and a complex auxiliary field $\left(F, G_{i}\right)$ as it can be seen from their expansions in the $\theta$ variables,

$\Psi=A(y)+\sqrt{2} \theta \chi(y)+\theta \theta F(y)$

$\Phi_{i}=B_{i}(y)+\sqrt{2} \theta \xi_{i}(y)+\theta \theta G_{i}(y)$.

Dynamics is governed by the Lagrangian density

$$
\begin{aligned}
L= & \int d^{4} \theta \Psi \Psi^{+}+\sum_{i=1}^{N} \int d^{4} \theta \Phi_{i} \Phi_{i}^{+} \\
& +\frac{g}{\sqrt{N}} \sum_{i=1}^{N} \int d^{2} \theta \Phi_{i} \Psi^{2} .
\end{aligned}
$$

The cubic superfield interaction corresponds to a Yukawa coupling between scalar and fermions and, after the elimination of the auxiliary fields, to a quartic coupling of the scalars. The Lagrangian reads in terms of component fields:

$$
\begin{aligned}
L= & i \partial_{\mu} \bar{\chi} \bar{\sigma}^{\mu} \chi+i \sum_{i=1}^{N} \partial_{\mu} \bar{\xi}_{i} \bar{\sigma}^{\mu} \xi_{i}+A^{*} \square A \\
& +\sum_{i=1}^{N} B_{i}^{*} \square B_{i}+F^{*} F+\sum_{i=1}^{N} G_{i}^{*} G_{i} \\
& +\frac{g}{\sqrt{N}} \sum_{i=1}^{N}\left(A^{2} G_{i}+2 A B_{i} F-\chi^{2} B_{i}-2 \xi_{i} \chi A+\text { h.c. }\right) .
\end{aligned}
$$

In our conventions (the same as in [8]), we have $\operatorname{diag} g_{m n}=$ $(-1,1,1,1)$ and

$\square=-\partial_{t}^{2}+\nabla^{2}$.

\section{Dyson-Schwinger equations}

We only consider corrections to the propagators of the fields associated to the superfield $\Psi$, as corrections to the propagation of the superfield $\Phi_{i}$ get a factor $1 / N$ and do not contribute in the large $N$ limit. Moreover supersymmetry ensures that there are no divergent vertex corrections so that what we are calculating represents effectively the order zero of the large $N$ development.

The set of diagrams that we consider is the same as the one studied in [6], except that now the diagrams have to be considered as superfield diagrams and therefore, each one will correspond to a sum of ordinary Feynman diagrams. The number of such diagrams grows rapidly with the number of loops and for example a given 12 loop superdiagram corresponds to 18144 ordinary diagrams.

The diagrams we are looking at correspond to the expansion of the Dyson-Schwinger equations graphically represented in Fig. 1.

With our (Minkowski) conventions, free propagators read

$$
\begin{aligned}
& \Pi_{0 A}^{-1}(p)=\left\langle 0\left|T\left(A(x) A^{*}\left(x^{\prime}\right)\right)\right| 0\right\rangle=i \square^{-1}\left(x-x^{\prime}\right), \\
& \Pi_{0 F}^{-1}(p)=\left\langle 0\left|T\left(F(x) F^{*}\left(x^{\prime}\right)\right)\right| 0\right\rangle=i \delta\left(x-x^{\prime}\right), \\
& \left(\Pi_{0 \chi}^{-1}\right)_{\alpha \dot{\beta}}(p)=\left\langle 0\left|T\left(\chi_{\alpha}(x) \bar{\chi}_{\dot{\beta}}\left(x^{\prime}\right)\right)\right| 0\right\rangle=\sigma_{\alpha \dot{\beta}}^{m} \partial_{m} \square^{-1}\left(x-x^{\prime}\right) .
\end{aligned}
$$

It will be convenient to write Schwinger-Dyson equations in Euclidean space. Then calling $\Pi_{F}, \Pi_{\chi}, \Pi_{A}$ the full propagators for fields $F, \chi$ and $A$ in (Euclidean) momentum space, the corresponding self-energies are introduced as

$\Pi_{A}^{-1}(p)=p^{2}\left(1-\Sigma_{A}\left(p^{2}\right)\right)$

$\Pi_{F}^{-1}(p)=-\left(1-\Sigma_{F}\left(p^{2}\right)\right)$,

$\Pi_{\chi}^{-1}(p)=p_{m} \sigma^{m}\left(1-\Sigma_{\chi}\left(p^{2}\right)\right)$.

We consider a simple one-loop correction to the self-energy, where one of the propagators has a momentum-dependent 
renormalization $\Sigma\left(p^{2}\right)$. The simplest case is the one for the self-energy of the auxiliary field $F$, since there are only contributions from a single diagram with scalar propagators:

$$
\Sigma_{F}\left(p^{2}\right)=-\frac{g^{2}}{4 \pi^{4}} \int d^{4} q \frac{1}{q^{2}\left(1-\Sigma_{A}\left(q^{2}\right)\right)(p-q)^{2}} .
$$

For the fermion $\chi$, we have the two contributions shown in Fig. 1,

$$
\begin{aligned}
p_{m} \sigma^{m} \Sigma_{\chi}\left(p^{2}\right) & \\
= & -\frac{g^{2}}{4 \pi^{4}}\left(\int d^{4} q \frac{q_{m} \sigma^{m}}{q^{2}\left(1-\Sigma_{\chi}\left(q^{2}\right)\right)(p-q)^{2}}\right. \\
& \left.+\int d^{4} q \frac{p_{m} \sigma^{m}-q_{m} \sigma^{m}}{q^{2}\left(1-\Sigma_{A}\left(q^{2}\right)\right)(p-q)^{2}}\right) .
\end{aligned}
$$

In the case of the scalar $A$, the three different terms shown in Fig. 1 evaluate as:

$$
\begin{aligned}
p^{2} \Sigma_{A}\left(p^{2}\right)= & \frac{g^{2}}{4 \pi^{4}}\left(\int d^{4} q \frac{-1}{q^{2}\left(1-\Sigma_{A}\left(q^{2}\right)\right)}\right. \\
& +\int d^{4} q \frac{-1}{\left(1-\Sigma_{F}\left(q^{2}\right)\right)(p-q)^{2}} \\
& \left.+\int d^{4} q \frac{-\operatorname{Tr}\left(q_{n} \bar{\sigma}^{n}\left(q_{m} \sigma^{m}-p_{m} \sigma^{m}\right)\right)}{q^{2}\left(1-\Sigma_{\chi}\left(q^{2}\right)\right)(p-q)^{2}}\right) .
\end{aligned}
$$

A consistent ansatz to solve these equations is to take

$\Sigma_{A}=\Sigma_{\chi}=\Sigma_{F}=\Sigma$.

Indeed, with this ansatz, the two integrals in (11) combine to give $p_{m} \sigma^{m}$ times the right-hand side of (10). Similarly, the equation for $\Sigma_{A}$ (12) becomes $p^{2}$ times (10). Note that $\operatorname{Tr}\left(\sigma_{n} \bar{\sigma}_{m}\right)=-2 g_{m n}$.

The integration in (10) can be done along the same lines as in [6]. The angular integration uses the fact that the angular average of $1 /(p-q)^{2}$ is $1 / \max \left(p^{2}, q^{2}\right)$. With the variables $x=p^{2}$ and $y=q^{2}$, the radiative correction becomes

$\Sigma(x)=-\frac{g^{2}}{4 \pi^{2}}\left[\int_{0}^{x} d y \frac{1}{x(1-\Sigma(y))}+\int_{x}^{\infty} \frac{1}{y(1-\Sigma(y))}\right]$.

Taking the $x$ derivative to get rid of the infinite constant, we arrive first at

$x^{2} \frac{d}{d x} \Sigma(x)=+\frac{g^{2}}{4 \pi^{2}} \int_{0}^{x} d y \frac{1}{(1-\Sigma(y))}$.

A further derivation allows to obtain a simple differential equation for $\Sigma$,

$(1-\Sigma(x)) D(D+1) \Sigma(x)=\frac{g^{2}}{4 \pi^{2}}=a$

with $D=x d / d x$, which is equivalent to $D=d / d \log \left(p^{2} / \mu^{2}\right)$.

\section{Anomalous dimension}

Eq. (16) is very similar to the one obtained for the Yukawa case in [6]. The only difference is that the operator $D(D+1)$ replaces in the present case the $D(D+2)$ one in [6]. The two equations can then be identified by rescaling the variable $L=$ $\log \left(p^{2} / \mu^{2}\right)$ and $a$ by appropriate factors. The results derived in [6] can then be reobtained taking into account the correct factors.

The most direct way of obtaining a solution of Eq. (16) is to solve for $\Sigma(x)$ order by order in $a$, using the renormalization constraint $\Sigma\left(\mu^{2}\right)=0$. At order $n$ in $a, \Sigma(x)$ is a polynomial in $L$ of order $n$ without constant terms. The renormalization group $\gamma$ function is then the first derivative in $L$ for $L=0$.

$\gamma(a)=\left.\frac{d \log \left(1-\Sigma\left(p^{2}, a\right)\right)}{d \log \left(p^{2}\right)}\right|_{p^{2}=\mu^{2}}$.

Here $\gamma$ is the anomalous dimension for all the fields in the scalar multiplet $\Psi$.

As was shown already in [6], this procedure can be further simplified if one directly computes $\gamma$ through the use of a nonlinear equation that can be deduced from Eq. (16). Indeed, consider the renormalization group relation [9]

$d\left(\frac{p^{2}}{\mu^{2}}, a\right)=d\left(\frac{p^{2}}{s^{2}}, a\left(s^{2}\right)\right) d\left(\frac{s^{2}}{\mu^{2}}, a\right)$,

where

$d\left(p^{2}, a\right)^{-1}=1-\Sigma\left(p^{2}, a\right)$.

Differentiation of Eq. (18) with respect to $p^{2}$ one obtains

$\frac{d \log (1-\Sigma)}{d \log p^{2}}=\gamma\left(a\left(s^{2}\right)\right)$

or

$\frac{d \log (1-\Sigma)}{d \log p^{2}}=\gamma\left(\frac{a}{(1-\Sigma)^{2}}\right)$.

This last identity was obtained as a consequence of the nonrenormalization of the vertex which implies a connection between the $\beta$ and $\gamma$ functions of the renormalization group. It should be noted that Eq. (21), which was derived here using the renormalization group, can also be derived using Hopf algebra arguments as explained in [6]. Indeed, in this last work the dependence of the self-energy on the momentum and the anomalous dimension (the propagator-coupling duality) based on the Hopf structure of Feynman diagrams is used to prove Eq. (21). Here, our derivation relies instead on the renormalization group relation Eq. (18).

Let us now rewrite Eq. (21) in the form

$D(1-\Sigma)=(1-\Sigma) \gamma\left(a /(1-\Sigma)^{2}\right)$

and apply the operator $(D+1)$ to both sides. After some work one ends with

$\gamma=-a+a^{2} \frac{d\left(\gamma^{2} / a\right)}{d a}$. 
From this nonlinear differential equation one can derive the perturbative expansion of $\gamma$,

$\gamma=\sum_{n>0} G_{n}(-a)^{n}$

$G_{n+1}=n \sum_{k=1}^{n} G_{k} G_{n+1-k}$.

A simple Mathematica program allows to evaluate $G_{n}$ for large values of $n$ according to this recurrence relation. As an example, $G_{100}$ can be evaluated quasi immediately giving

$G_{n}=242124042294816690391731848271216584637373707$

300511162111032048620842352993102243965346961

024316735330194465855125330800645887484858421

550031445472180813110312557469722336097340105

1786164.

We can then compute $\gamma$ as a function of $a$ using Eq. (24). However being $G_{n} \sim(2 n-1)$ !! for large $n$, the series is asymptotic and therefore we use a Padé-Borel resummation method. We start from the approximation

$\gamma \approx-a \int d x P(a x) \sqrt{x} \exp (-x)$,

where $P(x)$ is defined by the following series

$P(x)=\sum(-1)^{n} \frac{G_{n} x^{n-1}}{\Gamma(n+1 / 2)}$.

Numerical values for $\gamma$ can then be obtained using a Padé $[N \backslash M]$ approximant for $P(x)$. If one avoids the values of $[N \backslash M]$ for which this approximant has poles on the positive real axis, one can obtain stable evaluations for growing values of $a$. The successive approximations are fully coherent and higher degree approximants allow to reach higher values of the coupling constant $g, a=g^{2} / 4 \pi^{2}$. In a few seconds one can compute the anomalous dimension $\gamma$ for values $g^{2} \approx 700$ with a relative accuracy of the order of $10^{-10}$. As an example, one finds $\gamma\left(g^{2}=789.568\right)=-6.30706$ with a [99 $\left.\backslash 100\right]$ approximant. Rational solutions are not possible for such high orders and the precision afforded by floating point numbers is not sufficient to obtain solutions, but the use of higher precision allowed to reach easily this stage.

In Fig. 2, we show $\gamma$ as a function of $g^{2}$. Remarkably, we see from the numerical results that the anomalous dimension $\gamma$ of the scalar field can reach values where it exceeds the canonical one, so that, unexpectedly the propagator grows with the impulsion. One confirms this analytically from the asymptotic behavior of $\gamma$ for large $g$ that can be easily inferred from Eq. (23),

$\gamma \approx g \sqrt{\ln g}$.

We see that our calculation gives a complete knowledge of the renormalization group functions since, due to the nonrenormalization of the vertex one has $\beta=-2 \gamma$. In particular, the asymptotic behavior of $\gamma$ in Eq. (28) allows to prove that the coupling constant goes to infinity for a finite value of the scale.

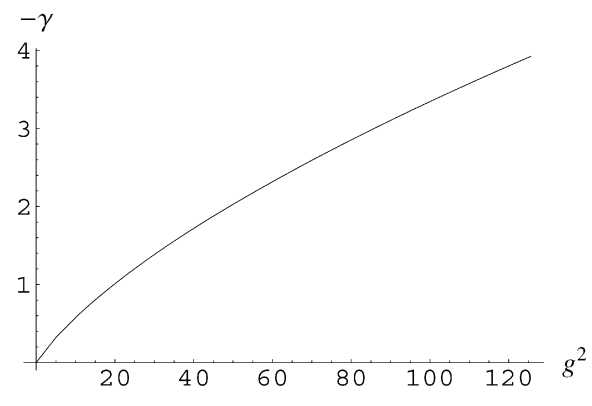

Fig. 2. Anomalous dimension as a function of $g^{2}$.

We end this section by noting that the renormalization group played a central rôle in obtaining the basic equations (21)-(23) from the Dyson-Schwinger equations. General information on the renormalization group have been presented in [4], deduced from the Hopf algebra structure of perturbative quantum field theory. It would be of interest to combine the two approaches to fully realize the potential of Dyson-Schwinger methods to obtain renormalization group functions.

\section{Discussion}

In this Letter we have generalized the calculations of [6] to a supersymmetric setting. We have chosen to study a WessZumino like model in which the vertex is not renormalized and this allowed us to calculate the order zero of the renormalization group functions in a large $N$ expansion in a closed form.

Writing the Dyson-Schwinger equations for propagators and proposing an appropriate ansatz for the corresponding selfenergies we have found a nonlinear differential equation for the anomalous dimension (Eq. (23)) from which one can derive a perturbative expansion for $\gamma$. Coefficients in such expansion can be very easily evaluated up to very large orders and a PadéBorel resummation allowed us to compute $\gamma(g)$ in a large range of coupling constants with great accuracy.

In our calculations, the power of the Hopf algebra approach has not be used explicitly. It is tempting to speculate, however, that the special features of perturbation theory in supersymmetric models should reflect in some particular structure within the Hopf algebra approach. We intend to discuss this issue in a future work.

\section{Acknowledgements}

We would like to thank the Sociedad Cientifica Argentina for hospitality. This work is partially supported by CONICET (PIP6160), ANPCyT (PICT 20204), CONICET/CNRS/PICS3172, UNLP, UBA and CICBA grants. M.B acknowledges CNRS support through his "mise à disposition".

\section{References}

[1] D. Kreimer, Adv. Theor. Math. Phys. 2 (1998) 303, q-alg/9707029.

[2] A. Connes, D. Kreimer, Commun. Math. Phys. 199 (1998) 203.

[3] A. Connes, D. Kreimer, Commun. Math. Phys. 210 (2000) 249, hep-th/ 9808042 . 
[4] A. Connes, D. Kreimer, Commun. Math. Phys. 216 (2001) 215, hep-th/ 0003188.

[5] D.J. Broadhurst, D. Kreimer, Phys. Lett. B 475 (2000) 63, hep-th/ 9912093.

[6] D.J. Broadhurst, D. Kreimer, Nucl. Phys. B 600 (2001) 403, hep-th/ 0012146.
[7] D. Kreimer, hep-th/0609004.

[8] J. Wess, J. Bagger, Supersymmetry and Supergravity, Princeton Univ. Press, Princeton, 1992.

[9] N.N. Bogoliubov, D.V. Shirkov, Introduction to the Theory of Quantized Fields, J. Wiley, New York, 1959 